\title{
Nuevas perspectivas sobre el movimiento en la filosofía francesa: la reivindicación ontológica de M. Merleau- Ponty y G. Deleuze
}

\section{( Jorge Nicolás Lucero}

Universidad de Buenos Aires -Université de Toulouse 2 Jean Jaurès / Consejo Nacional de Investigaciones

Científicas y Técnicas, Argentina

Directores: Dr. Esteban García, Dr. Pierre Montebello

Jurado: Dr. David Lapoujade, Dra. Aline Wiame, Dr. Andrés Osswald, Dra. Graciela Ralón

Fecha de defensa: 13 de septiembre de 2018

Aunque se trata de un concepto fundamental, el movimiento ha sido frecuentemente menospreciado como tema autónomo en la historia de la filosofía, especialmente tras el desarrollo de las ciencias físicas que se apropiaron de su estudio y lo redujeron a un estado no constitutivo de la materia. Esta tesis busca recuperar una indagación ontológica sobre el movimiento para mostrar su riqueza especulativa y relevancia para la filosofía contemporánea. Para ello, este trabajo centra sus análisis en las filosofías de Maurice Merleau-Ponty (1908-1961) y Gilles Deleuze (1925-1995).

$\mathrm{Al}$ abordar en clave ontológica el pensamiento de estos filósofos, esta investigación encuentra una reivindicación original sobre la cuestión, en donde el movimiento como proceso se opone no solamente a la concepción geométrica del movimiento, sino también al movimiento ontológico aristotélico. En un camino allanado por Bergson y por la fenomenología, ambos filósofos hacen posible un nuevo concepto de movimiento ontológico separado de aquellas nociones y de las modelizaciones antropomórficas, haciendo posible investigar la movilidad en sí misma y otorgar nuevas categorías para una filosofía de la naturaleza. Asimismo, la noción de movimiento que puede inferirse en ambas ontologías sostiene una primacía de este concepto por sobre los del espacio y el tiempo. Dicho de otro modo, el movimiento no es solamente un fenómeno espaciotemporal, sino un acto espacio-temporalizante.

La tesis comprende una introducción, tres partes principales de dos capítulos cada una, y una conclusión. La primera parte es un recorrido histórico acerca del tratamiento y la comprensión del movimiento en la conceptualización filosófica, así como el procedimiento y la forma que llevaron al movimiento de poseer una relevancia ontológica a su comprensión como un estado de la materia geométricamente mensurable sin ningún valor especulativo, para llegar a una rehabilitación filosófica del mismo en algunas filosofías de los siglos XIX y XX.

El primer capítulo comprende los avatares del movimiento hasta su matematización plena en la física newtoniana. Se comienza con una defensa heterodoxa de la figura de Zenón de Elea, en cuyas paradojas del movimiento se suele encontrar el nacimiento de la metafísica de la presencia. Las paradojas eleáticas ponen al descubierto no a un Zenón monista y defensor fiel de las tesis parmenídeas, sino a un Zenón nihilista, que coloca la racionalidad frente a sus límites en la búsqueda por comprender el cambio. Luego se analizan los rudimentos de la física aristotélica y el rol que ocupa la noción de movimiento, para así determinar cómo la definición de movimiento ontológico y sus tipos (desplazamiento, alteración, crecimiento y disminución) constituyen el pilar de la cosmología del Estagirita, sin obviar el modelo que él adopta para su argumentación, a saber, el deseo y la praxis humana. En lo siguiente, se describen los desarrollos de una mecánica medieval que busca independizar el movimiento local, lo que se consagra en la física de Galileo Galilei. Gracias a él, el movimiento local queda emancipado del esquema finalista al que era sometido en la física aristotélica; pero en este acto emancipador se observa, no solo una distancia radical con Aristóteles, sino también la destrucción de cualquier valoración ontológica para el movimiento. Aquí nace el movimiento como estado de la materia, sin transferencias de formas, sin lugares naturales, geometrizado y separado del reposo solamente por una diferencia cuantitativa. Este mismo paso se analiza en las variantes de René Descartes, quien afirma el movimiento como estado a partir de su concepción mecanicista de la naturaleza en general, e Isaac Newton, quien culmina la matematización del movimiento incluso apelando a los conceptos metafísicos de espacio, tiempo y movimiento absolutos. 
El capítulo 2 analiza la recuperación del valor especulativo del movimiento en el siglo XIX. Allí presta atención, como historia reciente de esta reivindicación, al bergsonismo y a la fenomenología, gracias a quienes el movimiento vuelve a arraigarse a la noción de vida. En cuanto a Bergson, quien rechaza cualquier reducción del movimiento a las mediciones geométricas y las repeticiones mecánicas por constituir la expresión de la duración, el movimiento pasará de mentar la duración como la esencia de la conciencia a constituir la duración en sentido ontológico, construyendo una noción de movilidad real carente de objetos móviles predeterminados. El trabajo enfatiza, sin embargo, la inconsistencia de la filosofía bergsoniana del movimiento por carecer de una teoría del espacio que supere a la noción geométrica, y que el propio filósofo encuentra problemática para comprender el ser como devenir. La fenomenología, por su parte, mediante las interpretaciones del movimiento en los trabajos de Edmund Husserl, Martin Heidegger y Jan Patočka, rescata la significación vital del movimiento. En el caso de Husserl, se describe el rol de las cinestesias que dan testimonio del "yo me muevo" originario que co-constituye el mundo lateralmente a la conciencia trascendental. Respecto de Heidegger y Patočka, se resalta la importancia que tiene para ambos la relectura de la Física de Aristóteles en la tematización de la naturaleza humana. Mientras que Heidegger se apropia del concepto para diezmarlo y diferenciarlo de la existencia humana, dado su lazo con la presencia que es incompatible con la temporalidad ex-tática del Dasein, Patočka verá en una kínesis desustancializada la raíz de los movimientos de la existencia humana y su vinculación con el mundo en la estructura del aparecer.

La segunda parte de la tesis es de carácter sistemático en la medida en que interpreta en cada uno de sus capítulos las filosofías de Merleau-Ponty y Deleuze como ontologías. El tercer capítulo presenta la filosofía merleau-pontyana como una ontología de la relación. Esta exégesis sostiene que Merleau-Ponty pertenece a un lugar limítrofe dentro de la corriente fenomenológica, la cual le permite depurar y rediseñar sus preocupaciones filosóficas pero que no las determina. Al contrario, el trabajo advierte que el descubrimiento de la Gestalt como herramienta teórica le facilitará la construcción de una ontología indirecta que colisiona tanto con la fenomenología husserliana como con la ontología fundamental de Heidegger. La ontología de Merleau-Ponty no queda articulada bajo la órbita de la reducción fenomenológica, sino bajo una adopción particular de la dialéctica, que presenta, siguiendo las palabras del filósofo, "el ser como paradoja”. Esta cuestión condujo a pensar la cuestión del sujeto como paradoja, de tal manera que se abre la pregunta por el campo trascendental como terreno de la indagación filosófica. La tesis sostiene que Merleau-Ponty inicia desde su primera obra una renovación de la filosofía trascendental donde se suprime el condicionamiento lógico-formal para invocar un "arreglo contingente" entre las partes del campo fenomenal. Así, el trascendental queda infestado de elementos empíricos que deslegitiman la afirmación de cualquier a priori y resaltan la dimensión de la génesis. De ello resulta la supresión de cualquier operatividad de la distinción entre lo trascendental y lo empírico. Dicha distinción se ve remplazada por la noción de institución y el inicio de una nueva reflexión sobre el concepto de naturaleza.

El cuarto capítulo defiende la importancia de la ontología en la obra de Deleuze a través del problema de la distribución. Preocupado por las relaciones entre el ser y el pensamiento, y buscando superar la relación dada por la representación, Deleuze propone una ontología unívoca que resignifica la distribución de lo real según un principio inmanente. Gracias a este principio, el ser y el devenir se enlazan por vía de la repetición, instalando un sentido problemático que lo vuelve el eventum tantum de todos los acontecimientos. Asimismo, la distinción modal entre lo virtual y lo actual concierne a esta nueva relación entre ser y pensamiento al punto de reformar de manera novedosa el concepto mismo de Idea, a través de la cual se inmiscuye, de manera necesaria, la purificación deleuziana del campo trascendental. Este trabajo sostiene que el filósofo ha sabido llevar el trascendental hasta sus últimas consecuencias, agotando su funcionalidad y reemplazándola por la noción de plano de consistencia, sin que ello degrade la preocupación ontológica; por el contrario, la afirma como ontología práctica o distribución del ser.

La Tercera Parte desarrolla la problemática del movimiento dentro de estas dos ontologías. El propósito general de los capítulos que la componen consiste en mostrar la construcción de un movimiento ontológico distinta a la presentada por la física aristotélica, atendiendo a los siguientes puntos: el a-teleologismo del movimiento; la utilización de un esquema conceptual diferente al binomio potencia y acto; las alusiones a modelos biológicos no-humanos; y la crítica a una primacía de la espacialidad como condición del movimiento.

El quinto capítulo prueba que el movimiento es un fenómeno bisagra dentro de la ontología de Merleau-Ponty, pues gracias a este se hace comprensible la noción de carne. Se destaca en este análisis 
la influencia esencial del psicólogo Max Wertheimer para comprender la organización endógena del campo fenomenal manifestada radicalmente en el movimiento, y la cual aleja al fenómeno de la interpretación del Estagirita, por tratarse de un movimiento constituido según un modelo no-antropomórfico. Además del gestaltismo, el trabajo profundiza sobre otro modelo con el cual Merleau-Ponty supo revertir la relación de fundamentación del movimiento respecto del espacio y el tiempo: el cine. Tras esta profundización, la tesis analiza finalmente el vínculo entre esa movilidad primigenia y una inmovilidad fundamental al interior de la noción de carne, vínculo que aparece con el concepto de inminencia.

El capítulo 6 defiende la importancia del problema del movimiento en la totalidad de la obra deleuziana, en contra de las interpretaciones que la circunscriben a sus libros sobre el cine. Aunque de manera mayormente implícita, el movimiento se presenta como la caracterización misma del ser ya que constituye la distribución creadora de lo real. En un primer tiempo se relevan las nociones de movimiento real y movimiento forzoso al momento de caracterizar la esencia de la repetición. Continuando las consecuencias de esta exposición, el despliegue de la noción de devenir hará visible una teoría de la movilidad pura que no recae en las dificultades bergsonianas y que hace de la velocidad un concepto metafísico y no una variable matemática. Por último, una descripción de la ontología de la imagen que Deleuze encuentra en el cine asienta la consistencia y coherencia de las nociones de imagen-movimiento e imagen-tiempo respecto de la noción de movimiento real. La noción de imagentiempo no responde simplemente a una inversión de la subordinación entre el tiempo y el movimiento, sino que la pretendida liberación del tiempo es, antes que nada, la liberación del movimiento como movimiento aberrante.

En las conclusiones, además de explicitar algunas cercanías entre ambos filósofos, se consideran los resultados de los capítulos anteriores en el contexto de dos filosofías contemporáneas: la fenomenología de Renaud Barbaras y la corriente del nuevo realismo. Por un lado, la filosofía barbarasiana, que desarrolla la noción de movimiento profundizando y reformando el trabajo de Patočka y Merleau-Ponty, no solo sostiene al deseo como esencia del movimiento de aparecer del sujeto, sino que también, postulando un movimiento del mundo de donde el sujeto emerge, este movimiento continúa teniendo como modelo al deseo. A diferencia de lo que ocurre en la fenomenología barbarasiana, la tesis argumenta que, no solo en Deleuze, sino incluso en Merleau-Ponty, el deseo deriva del movimiento y no es su esencia. En cuanto a los nuevos realismos, el trabajo intenta probar que su desarrollo previo evade la crítica al correlacionismo de Quentin Meillassoux y, más aún, que ambas nociones de movimiento desarrolladas superan la condición del "acceso humano" sin caer en el recurso a la objetualidad que propone la filosofía orientada a objetos como la de Graham Harman. 